\title{
Celiac Artery Compression Syndrome: An Overlooked Cause of Abdominal Pain
}

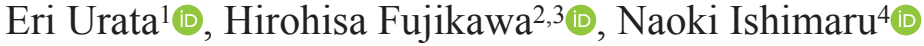 \\ ${ }^{1}$ Department of Internal Medicine, Suwa Central Hospital, Nagano, Japan \\ ${ }^{2}$ Department of Medical Education Studies, International Research Center for Medical Education, Graduate School of Medicine, The University of Tokyo, \\ Tokyo, Japan \\ ${ }^{3}$ Department of Internal Medicine, Suwa Central Hospital, Nagano, Japan \\ ${ }^{4}$ Department of Surgery, Suwa Central Hospital, Nagano, Japan
}

A 27-year-old female with depression presented to the hospital with a 4-year history of postprandial epigastric pain. One year ago, she had been diagnosed with psychogenic abdominal pain because of exhibiting no remarkable abnormalities on various examinations, including blood tests, abdominal computed tomography (CT), and upper gastrointestinal endoscopy. On physical examination, body mass index was 18.3 . The abdomen was soft, and there was moderate epigastric discomfort on deep palpation. Abdominal auscultation detected a bruit in the epigastrium, which was exacerbated during deep expiration. On performing abdominal Doppler ultrasonography (US), the peak systolic velocity of the celiac artery (CA) was found to be elevated $(256 \mathrm{~cm} / \mathrm{s})$ during deep exhalation (Figure 1a). Contrast-enhanced CT demonstrated CA stenosis with a typical hook-shaped appearance (Figure 1b). Thus, the patient was diagnosed with CA compression syndrome (CACS); subsequently, laparoscopic surgical intervention was performed with the patient under general anesthesia. The lesser omentum was divided. The common hepatic artery and the left gastric artery were identified and followed to the origin of the celiac trunk. The median arcuate ligament that was compressing the celiac artery was resected (Figure 1c). Her abdominal pain improved postoperatively, and follow-up imaging demonstrated decompression of the celiac axis (Figure 1d and 1e). The patient's consent was obtained.
CACS, also known as median arcuate ligament syndrome or Dunbar Syndrome, is an uncommon disorder caused by stenosis of the CA. The primary pathological mechanism responsible for this disorder is external compression of the celiac trunk by the median arcuate ligament. ${ }^{1}$ During expiration, the diaphragm moves cranially, causing stretching of the diaphragmatic crura, which leads to more compression of the celiac axis. ${ }^{2}$ The typical clinical presentation involves the following clinical triad in young, thin, adult females: postprandial epigastric pain, weight loss, and a bruit in the epigastric region. ${ }^{3}$ Beneficial diagnostic modalities include Doppler US, CT angiography, and magnetic resonance angiography. ${ }^{4}$ Although surgical division of the median arcuate ligament may be effective in relieving the symptoms, optimal treatment of CACS remains an area of controversy. ${ }^{3}$ Thus, shared decision making about treatment options with the patient is important.

CACS is often overlooked due to the lack of awareness of the disease among physicians and its nonspecific symptoms. This condition may be misdiagnosed as psychogenic abdominal pain, especially in patients with a history of psychiatric disorders, as in the case presented here. However, in CACS patients, stenosis of the CA reduces blood supply to the celiac territory. Moreover, the intraluminal pressure difference between the CA and the superior mesenteric artery increases compensatory blood flow to the pancreaticoduodenal arcade, possibly resulting in pancreaticoduodenal artery aneurysms,

Corresponding author: Hirohisa Fujikawa, Department of Medical Education Studies, International Research Center for Medical Education, Graduate School of Medicine, The University of Tokyo, Tokyo, Japan

e-mail: hirohisa.fujikawa@gmail.com

Received: September 24, 2021 Accepted: November 11, 2021 Available Online Date: January 18, 2022 • DOI: 10.4274/balkanmedj.galenos.2021.2021-9-100

Available at www.balkanmedicaljournal.org

ORCID iDs of the authors: E.U. 0000-0002-1503-8197; H.F. 0000-0002-8195-1267; N.I.; 0000-0001-8265-3627.

Cite this article as:

Urata E, Fujikawa H, Ishimaru N. Celiac Artery Compression Syndrome: An Overlooked Cause of Abdominal Pain. Balkan Med J.; 2022; $39(1): 71-2$.

Copyright@Author(s) - Available online at http://balkanmedicaljournal.org/ 

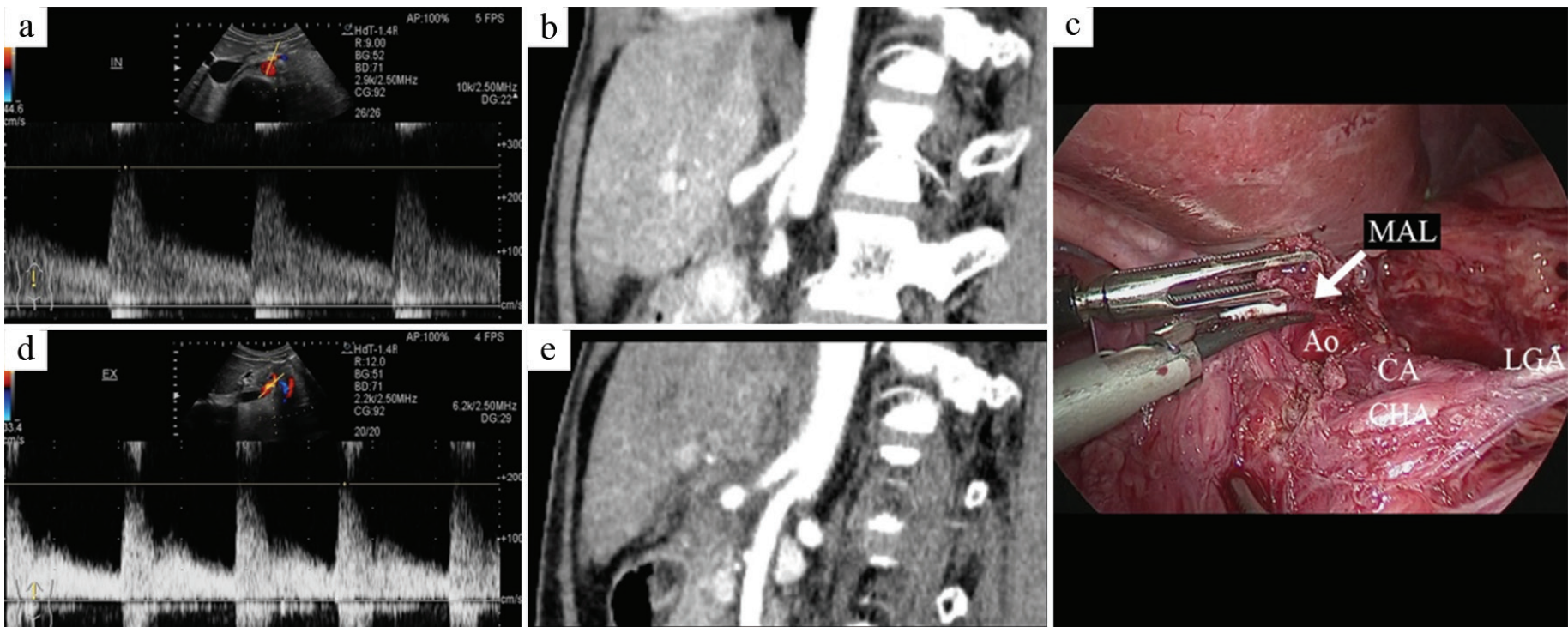

FIG 1 a-e. (a) Abdominal Doppler ultrasound showed elevated peak systolic velocity $(256 \mathrm{~cm} / \mathrm{s})$ at the origin of the celiac artery during deep expiration. (b) Contrast-enhanced abdominal computed tomography revealed celiac artery stenosis with a typical hook-shaped appearance. (c) Laparoscopic surgical resection of the median arcuate ligament was conducted (Ao, aorta; CA, celiac artery; CHA, common hepatic artery; LGA, left gastric artery; MAL, median arcuate ligament). (d and e) Postoperative abdominal imaging modalities showed decompression of the celiac trunk (d, Doppler ultrasound; E, contrast-enhanced computed tomography).

which may rupture and lead to hemorrhagic shock. ${ }^{5}$ Therefore, early diagnosis is crucial and may improve patient prognosis.

Patient Consent for Publication: Written informed consent was obtained from the patient.

Author Contributions: Concept - E.U., H.F., N.I.; Supervision - H.F.; Data Collection and/or Processing - E.U., H.F., N.I.; Literature Review - E.U., H.F., N.I.; Writing - E.U., H.F., N.I.

Conflict of Interest: The authors has no conflict of interest to declare.

\section{REFERENCES}

1. Goodall R, Langridge B, Onida S, Ellis M, Lane T, Davies AH. Median arcuate ligament syndrome. J Vasc Surg. 2020;71:2170-2176. [CrossRef]
2. Reuter SR, Bernstein EF. The anatomic basis for respiratory variation in median arcuate ligament compression of the celiac artery. Surgery. 1973;73:381-385. [CrossRef]

3. Kim EN, Lamb K, Relles D, Moudgill N, DiMuzio PJ, Eisenberg JA. Median Arcuate Ligament Syndrome-Review of This Rare Disease. JAMA Surg. 2016;151:471-477. [CrossRef]

4. Sultan S, Hynes N, Elsafty N, Tawfick W. Eight years experience in the management of median arcuate ligament syndrome by decompression, celiac ganglion sympathectomy, and selective revascularization. Vasc Endovascular Surg. 2013;47:614-619. [CrossRef]

5. Kallamadi R, deMoya M, Kalva S. Inferior pancreaticoduodenal artery aneurysms in association with celiac stenosis/occlusion. Semin Intervent Radiol. 2009;26:215-223. [CrossRef] 